\title{
NT5E CpG island methylation is a favourable breast cancer biomarker
}

\section{Lo Nigro',10, M Monteverde ${ }^{1,10}$, S Lee ${ }^{2,10}$, L Lattanzio', D Vivenza', A Comino ${ }^{3}$, N Syed ${ }^{4}$, A McHugh $^{5}$, H Wang ${ }^{5}$, C Proby ${ }^{5}$, O Garrone ${ }^{6}$, M Merlano $^{6}$, E Hatzimichael ${ }^{7}$, E Briasoulis $^{7}, \mathrm{O} \mathrm{Gojis}^{8}, \mathrm{C} \mathrm{Palmieri}^{8}$, L Jordan', P Quinlan', A Thompson' and T Crook ${ }^{*, 9}$}

'Laboratory of Cancer Genetics and Translational Oncology, Oncology Department, S. Croce General Hospital, Cuneo, Italy; ${ }^{2}$ Immunobiology Laboratory, London Research Institute, Lincoln's Inn Fields Laboratories, 44 Lincoln's Inn Fields, London, UK; ${ }^{3}$ Department of Pathology, S. Croce General Hospital, Cuneo, Italy; ${ }^{4}$ Faculty of Medicine, Imperial College London, Neuroscience Centre, Charing Cross Hospital, London, UK; ${ }^{5}$ Wellcome Trust Centre for Molecular Medicine, Division of Medical Sciences, Clinical Research Centre and Department of Dermatology, Ninewells Hospital, University of Dundee, Dundee DDI 9SY, UK; ${ }^{6}$ Oncology Department, S. Croce General Hospital, Cuneo, Italy; ${ }^{7}$ Interscience Molecular Oncology Laboratory, University of loannina Cancer Biobank Center, loannina, Greece; ${ }^{8}$ Cancer Research UK Laboratories, Division of Cancer, Imperial College London-Hammersmith Campus, Du Cane Road, London, UK; ${ }^{9}$ Division of Cancer Research, Medical Research Institute, University of Dundee, Ninewells Hospital and Medical School, Dundee DDI 9SY, UK

BACKGROUND: Relapse risk assessment and individual treatment recommendations remain suboptimal for breast cancer patients. In the light of existing preclinical and clinical data, we studied NT5E (5'-nucleotidase, ecto) expression and NT5E CpG island methylation in breast cancer.

METHODS: We used RT-PCR, qPCR, methylation-specific PCR and pyrosequencing to analyse NT5E in breast carcinoma cell lines and primary and breast carcinomas.

RESULTS: NT5E CPG island methylation was inversely associated with NT5E expression in breast carcinoma cell lines. In clinical series, patients whose primary tumours had NT5E CpG island methylation were less likely to develop metastasis $(P=0.003, O R=0.34,95 \%$ Cl: 0.17-0.69). In 3/4 paired samples, NT5E was methylated in primary tumours and demethylated in CNS metastases. Patients progressing to non-visceral as compared with visceral metastases were more likely to have NT5E CpG island methylation in primary tumours $(P=0.01, O R=||$ I.8). Patients with tumours lacking detectable methylation had shorter disease-free survival (DFS) $(P=0.00 \mathrm{I}, \mathrm{HR}=2.7)$ and overall survival $(\mathrm{OS})(P=0.00 \mathrm{I}, \mathrm{HR}=3)$. The favourable prognostic value of NT5E methylation was confirmed in oestrogen receptor negative $(P=0.01 \mathrm{I}, \mathrm{HR}=3.27,95 \% \mathrm{Cl}: 1.3 \mathrm{I}-8.12)$ and in triple negative cases $(P=0.004$; $H R=6.2,95 \% \mathrm{Cl}:$ 1.9-20). Moreover, we observed a more favourable outcome to adjuvant chemotherapy in patients whose tumours were positive for NT5E CPG island methylation: DFS $(P=0.0016, \mathrm{HR}=5.1,95 \% \mathrm{Cl}: 1.8-\mid 4.37)$ and $\mathrm{OS}(P=0.0005$, $\mathrm{HR}=7.4,95 \% \mathrm{Cl}: 2.416-23.08)$.

CONCLUSION: NT5E CPG island methylation is a promising breast cancer biomarker.

British Journal of Cancer (2012) 1 07, 75-83. doi:I0.1038/bjc.2012.212 www.bjcancer.com

Published online 31 May 2012

(c) 2012 Cancer Research UK

Keywords: NT5E; breast cancer; metastasis; epigenetics; triple-negative breast cancer

Prognostic and predictive factors used to inform optimal treatment approaches for individual breast cancer patients have gradually moved over the last three decades from clinico-pathological features defined by mathematical probabilistic approaches towards biological and eventually genetic biomarkers (Perou et al, 2000; Paik et al, 2004; Andreopoulou and Hortobagyi, 2008). Current state-of-the-art approaches that take into consideration oestrogen receptor (ER), progesterone receptor and HER-2 expression in primary tumours advanced, albeit modestly, personalised breast cancer therapy. However, numerous patients continue to derive little benefit from their treatment (Korde and Gralow, 2011; Perez, 2011). Recently, the introduction of microarray-based gene

\footnotetext{
*Correspondence: Dr T Crook, Email: tr.crook@gmail.com

${ }^{10}$ These authors contributed equally to this work.

Received 3 January 2012; revised 15 March 2012; accepted 23 April

2012; published online 31 May 2012
}

expression refreshed the prospects for more accurate personalised approaches (Sotiriou et al, 2003; Roukos et al, 2007). However, relapse risk profiling and thereof treatment recommendations still remain suboptimal. Existing microarray-based gene-expression profiling tests are only slow progressing in overtaking classical markers as more accurate prognostic systems and predictors of response to specific therapeutics and are not yet established in clinical practice (Sotiriou and Pusztai, 2009).

Translational cancer research has recently started focusing on epigenetics as a novel and rich source of potential biomarkers (Visvanathan et al, 2006). In this context, methylation markers appear particularly promising for being cost-effective, biologically plausible and technically straightforward, which conform to suggested principles for breast cancer biomarker evaluation (McGuire, 1991).

NT5E (5'-nucleotidase, ecto) is located at 6q14-q21 (NC_0000 06.11) and encodes CD73, a plasma membrane protein that 
catalyses the conversion of extracellular nucleotides to membranepermeable nucleosides (Boyle et al, 1988). CD73 belongs to the $5^{\prime}$-nucleotidase family that have diverse physiological roles, especially in controlling nucleotide kinetics (Zimmermann, 1992). Evidence from a number of experimental systems suggests that expression of NT5E/CD73 may be important in increasing the invasive and metastatic properties of cancers and studies have shown that overexpression of NT5E may contribute to progression of breast cancer via generation of adenosine nucleoside (Zhou et al, 2007a; Zhou et al, 2007b). In breast cancer cell lines, NT5E overexpression results in an aggressive breast cancer phenotype and ectopic expression of NT5E in MCF-7 cells promotes cell viability, cell cycle progression and tumourigenesis (Wang et al, 2008). Conversely, depletion of NT5E mediated by inhibitory RNA causes reduction in cell growth rate, G0/G1 arrest and increased apoptosis in the triple-negative MDA MB 231 breast carcinoma cell line (Zhi et al, 2010). Moreover, animal models of cancer support a critical function for NT5E in mediating metastasis and hindering antitumour immunity (Stagg et al, 2011).

Given the proposed involvement of NT5E in malignant progression and apoptosis (Ujhazy et al, 1996; Mikhailov et al, 2008) there have been few translational studies of NT5E in breast cancer (Kruger et al, 1991; Spychala et al, 2004). In the latter study it was suggested that NT5E is suppressed by ER $\alpha$, but little else is known about the mechanisms regulating expression of NT5E either in breast cancer or more generally in neoplasia.

Methylation-dependent transcriptional silencing is a key genome regulatory mechanism, crucial for organism development and cellular differentiation, by which gene promoters are repressed impeding the transcription of dependent genes and thereof the production of encoded proteins (Jaenisch and Bird, 2003). Characteristic gene promoter methylation/demethylation occurs in cancer, which can involve either cancer-promoting or tumoursuppressing genes, and may serve as novel cancer biomarkers (Lopez et al, 2009). Here, we assessed the DNA methylation status of the NT5E CpG island in breast cancer and investigated for clinical relevance.

\section{MATERIALS AND METHODS}

\section{Cell lines}

Fourteen breast carcinoma cell lines (MDA MB 231, MDA MB 361, MDA MB 436, MDA MB 453, MDA MB 468, MCF-7, GI101, T47D, MCF12A, ZR75.1, MB 157, NCI, BT20 and CAL51) and a human mammary epithelial cell line were grown as described previously (Shah et al, 2009). Genomic DNA was extracted from cell pellets using the DNeasy Mini kit (Qiagen, Crawley, UK) according to the manufacturer's instructions. Total RNA was extracted using Trizol (Invitrogen, Paisley, UK). cDNA was synthesised from $1 \mu \mathrm{g}$ total RNA using oligo (dT) primers and the ImProm-II reverse transcriptase kit (Promega, Southampton, UK). For demethylation, cells were treated with $5 \mu \mathrm{m} 5^{\prime}$ azacytidine ( $5^{\prime}$ AZA; Sigma, St Louis, MO, USA) for 7 days. The cells were split every 2-3 days with the addition of fresh drug. After drug treatment, cells were harvested for $\mathrm{qPCR}$.

\section{Clinical material}

The study was performed with local ethical committee approval. NT5E was analysed in three independent breast cancer clinical series.

- Eighty-three predominantly ER-positive primary breast carcinomas from northern Italy.

- Twenty-three surgically resected, histologically confirmed CNS metastases from patients with breast carcinomas. In four patients, paired primary cancers were available and also analysed.
- One hundred and fifty-seven primary breast carcinomas from Tayside, Scotland; of which, 119 were ER positive, 11 were HER-2 positive only and 26 were triple negative breast cancer (TNBC).

In series I and III, cancers were randomly selected from the tissue archives and only included in the study following confirmation by an expert, specialist breast pathologist of (i) original diagnosis (ii) sufficient neoplastic cell representation. Cases from series II (CNS metastases) were identified by searching the neuropathology archives for cases of resected space occupying lesions. Cases were confirmed by histopathology to be metastatic breast carcinomas and tumour cell representation was again verified by histopathology. In series I and series III, we investigated the effect of NT5E CpG island methylation on risk of future relapse with metastatic disease and (for series III) the effect on clinical outcome. In series II, we analysed the frequency of NT5E CpG island methylation in metastatic breast carcinomas and for a subset of the cases, we compared methylation in primary and metastatic lesions. In all cases, expression of ER, PgR and HER-2 was determined according to normal protocols of clinical care. Staging and clinical follow-up were done according to standard clinical guidelines in each institution, typically with 3 monthly follow-up post-surgery and imaging (mammography and CT scans) where indicated, according to local guidelines. In series I and III, patients were treated adjuvantly according to normal clinical protocols. ER-positive patients were treated adjuvantly with endocrine therapy according to clinical guidelines at the time of treatment. This was typically with tamoxifen for 5 years. Isolation of genomic DNA was using Proteinase $\mathrm{K}$ for the formalinfixed, paraffin-embedded cases (series I and II) and as described previously for series III (Shah et al, 2009).

For statistical purposes, patients were grouped to those with non-visceral metastases, identified by bone-confined metastatic disease, lymph node only and bone + lymph node and those with visceral metastatic disease identified by brain, lung, liver and cutaneous metastases.

\section{Methylation analysis}

We identified a CpG island in the $5^{\prime}$ regulatory sequences of NT5E (http://www.genome.ucsc.edu/cgi-bin/hgGateway) and tested possible association between aberrant methylation in the CpG island and downregulation of mRNA expression using methylationspecific PCR (MSP) and pyrosequencing. DNA (0.5 $\mu \mathrm{g})$ was modified by sodium bisulphite using the Zymo EZ DNA Methylation kit (Zymo Research, Irvine, CA, USA). This process converts unmethylated cytosine residues to uracil, whereas methylated cytosine residues remain unchanged. Bisulphitemodified DNA was used as a template for methylation-specific PCR and pyrosequencing. CpGenome Universal Methylated DNA (Zymo Research) and normal human unmethylated DNA were used as positive and negative controls, respectively, in each experiment. Primers for methylation-specific PCR:

M forward primer: $5^{\prime}$-TATTTTATGAACGTTTTGCGTTACG-3'

M reverse primer: $5^{\prime}$-CTAAACTTACCACACTCTACCATCCG- $3^{\prime}$

U forward primer: $5^{\prime}$-ATTTTATGAATGTTTTGTGTTATGA- $3^{\prime}$

U reverse primer: $5^{\prime}$-AACTTACCACACTCTACCATCCACT- $3^{\prime}$

Each PCR was performed at least three times and scored independently by two observers.

Primers for pyrosequencing amplified a 170-bp fragment from the NT5E CpG island.

Sequences were as follows:

PCR F 5'-GTATTAGGGTATTATTTGGTTTAT-3'

PCR R 5'- BIOT -CTTACCACACTCTACCATCC- $3^{\prime}$

Polymerase Chain Reaction conditions were: $95^{\circ} \mathrm{C}$ for $10 \mathrm{~min}$, $95^{\circ} \mathrm{C}$ for $30 \mathrm{~s} / 54^{\circ} \mathrm{C}$ for $30 \mathrm{~s} / 72^{\circ} \mathrm{C}$ for $40 \mathrm{~s}$ for 40 cycles, $72{ }^{\circ} \mathrm{C}$ for $7 \mathrm{~min}$. PCR products were resolved on $2 \%$ agarose gels, visualised 
using a transilluminator, then analysed by pyrosequencing (Biotage Sample Prep kit, using forward primer). Analysis of \% methylation at each $\mathrm{CpG}$ dinucleotide was performed using $\mathrm{CpG}$ Software (Qiagen). Placental DNA was used as negative control of methylation ( $0 \%$ average methylation) and a commercial methylated DNA (Millipore, Waltford, UK) was used as positive control (98\% average methylation). The RasL12 CpG island was analysed using bisulphite sequencing and methylation-specific PCR. Location of primers for methylation-specific PCR and bisulphite sequencing is shown in Figure 7.

\section{NT5E expression analysis}

For qPCR analysis of expression, total RNA was isolated using the RecoverAll Total Nucleic Acid Isolation (Ambion, Austin, TX, USA). Twenty-five microlitre PCR reactions were performed using $50 \mathrm{ng}$ of cDNA obtained by reverse transcription. Amplification and analysis were done according to the manufacturer's protocol in 96-well plates in an ABI PRISM 7000 Sequence Detection System (Applied Biosystems, Foster City, CA, USA) and the pre-cast 'TaqMan Gene Expression Assays' (Applera, https://products.appliedbiosystems.com/) for NT5E (Hs001573922_m1). Quantification of the target transcript was performed in comparison to the reference transcript $\beta 2$ microglobulin (Hs99999907_m1), using the 'delta-delta Ct method' for comparing relative expression results in real-time PCR as outlined by PE Applied Biosystems (Perkin Elmer, Foster City, CA, USA).

\section{Statistics}

CpG island methylation status and presence or metastatic profile were assessed for associations using Fisher's exact test. Kaplan-Meier curves were used to estimate the probabilities of overall survival (OS) and disease-free survival (DFS) and the log-rank test to assess the statistical significance of differences in event rates. All statistical analyses were performed using Prism 5 (GraphPad software, Inc., La Jolla, CA, USA). Overall survival was defined as time from treatment start to death from any cause, or last follow-up date. Disease-free survival was defined as time from treatment start to disease progression or death for any cause. Living patients without evidence of progression were censored at the last follow-up. Analysis of the risk of death for the 157 tumours from Tayside was conducted using Cox proportional-hazards regression modelling. We examined the relationship between OS and the methylation status of the NT5E CpG island and known clinico-pathological parameters, including tumour size, tumour grade, ER status, PgR status, nodal status and HER-2.

\section{RESULTS}

\section{NT5E expression is regulated epigenetically in breast cancer}

Methylation in the NT5E CpG island was detectable in MDA MB 361, MDA MB 436, MDA MB 453, MCF-7, GI101, T47D, MCF12A and ZR75.1 cell lines (Figure 1A). By methylation-specific PCR, methylation was complete (no detecteable amplification in the U-specific PCR) in MDA MB 453 and T47D and partial in the remaining cell lines (amplification of both $U$ - and M-specific PCR). There was no detectable methylation in MDA MB157, MDA MB 231, MDA MB 468, NCI, BT20 and CAL51 (Figure 1A). To validate and extend these results, we performed pyrosequencing analysis of the NT5E CpG island in breast cancer cell lines. These included a subset of those analysed by methylation-specific PCR, together with additional lines not previously analysed (Figure 1B). Average methylation in the CpG island was $94 \%$ in MDA MB 453 and $88 \%$ in T47D, consistent with methylation-specific PCR (Figure 1B). There was partial methylation in MDA MB 436 (35\%) and MCF-7
(26\%) again consistent with methylation-specific PCR. MDA MB 231 and MDA MB 468 had average methylation levels of $2 \%$, also in agreement with methylation-specific PCR (summarised in Table 1). Next, we assessed expression of NT5E mRNA using RTPCR and correlated this with methylation status. These data (Figure 1, Table 1) imply a close correlation between methylation in the NT5E CpG island and absent/downregulated expression of NT5E mRNA. To further test this association, we used qPCR to analyse mRNA levels, initially in normal breast cells and cell lines with $\mathrm{CpG}$ island methylation. Expression was undetectable in MDA MB 453 and T47D (lines with almost complete methylation of the

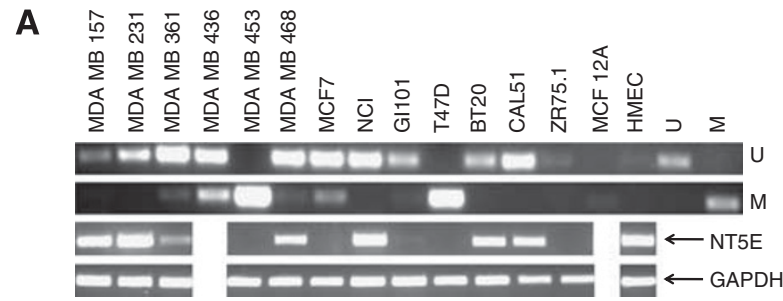

B

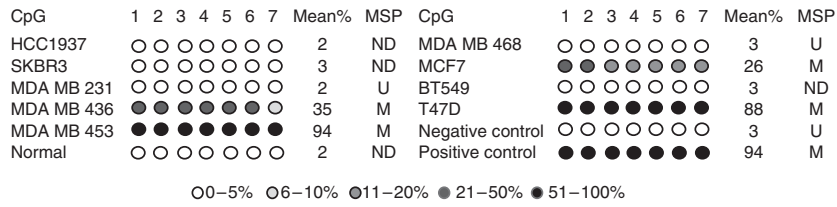

Figure I Methylation-dependent transcriptional silencing of NT5E in breast cancer cell lines. (A) Methylation in the NT5E CpG island correlates with downregulation of mRNA expression. The figure shows MSP analysis of the NT5E CpG island (upper panels) and semi-quantitative RT-PCR analysis of NT5E mRNA and the control gene GAPDH in breast carcinoma cell lines (bottom panels). MSP was performed as described in Materials and Methods. The figure shows unmethylated $(U)$ and methylated $(M)$ reactions for each cell line DNA. Also shown are control $U$ and $M$ DNA samples modified in parallel with the cell line DNA samples. (B) Pyrosequencing analysis of the NT5E CpG island in breast carcinoma cell lines. Pyrosequencing was done as described in Materials and Methods. The level of methylation is represented by the intensity of shading in the circles, each of which represents an individual CpG dinucleotide in the amplified fragment. The mean \% CpG methylation in the amplified fragment, together with the methylation-specific PCR analysis is also shown. Abbreviation: $\mathrm{ND}=$ not determined.

Table I Methylation in the NT5E CpG island in breast carcinoma cell lines

NT5E NT5E (pyro-

\begin{tabular}{|c|c|c|c|c|c|c|}
\hline Cell line & Phenotype & ER & PgR & HER-2 & (MSP) & $\begin{array}{l}\text { sequencing } \% \text { ) } \\
\text { segro }\end{array}$ \\
\hline MDA MB 157 & (Basal) & - & - & $\mathrm{N}$ & U & ND \\
\hline MDA MB 231 & (Basal) & - & - & $N$ & $U$ & 2 \\
\hline MDA MB 361 & (Luminal) & + & - & Amp & $U / M$ & ND \\
\hline MDA MB 436 & (Basal) & - & - & $N^{r}$ & $U / M$ & 35 \\
\hline MDA MB 453 & (Luminal) & - & - & Amp & $M$ & 94 \\
\hline MDA MB 468 & (Basal) & - & - & $N^{\top}$ & U & 2 \\
\hline MCF-7 & (Luminal) & + & + & N & $U / M$ & 26 \\
\hline MCFI2A & (Basal) & - & - & N & $U / M$ & ND \\
\hline GlIOI & (Basal & + & - & N & $U / M$ & ND \\
\hline T47D & (Luminal) & + & + & N & $M$ & 88 \\
\hline BT20 & (Basal) & - & - & N & U & ND \\
\hline CAL5I & (Basal) & - & - & N & $U$ & ND \\
\hline BT549 & (Basal) & - & - & $N$ & ND & 3 \\
\hline ZR75.I & (Luminal) & + & - & $\mathrm{N}$ & $\mathrm{U} / \mathrm{M}$ & ND \\
\hline HCCI937 & (Basal) & - & - & N & ND & 2 \\
\hline SKBR3 & (Luminal) & - & - & Amp & ND & 3 \\
\hline
\end{tabular}

Abbreviations: $\quad A m p=$ Amplified; $\quad E R=$ oestrogen receptor; $M=$ methylated; $M S P=$ methylation-specific $\quad P C R ; \quad N D=$ not determined; $P g R=$ progesterone receptor; $U=U$ nmethylated. Data on cell line phenotypes, ER, PgR and Her-2 are from Mackay et al (2009). 
A

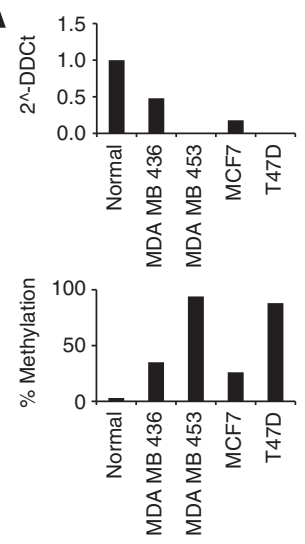

C

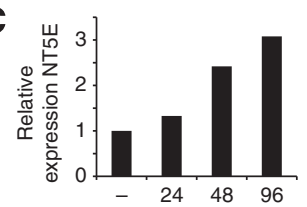

B

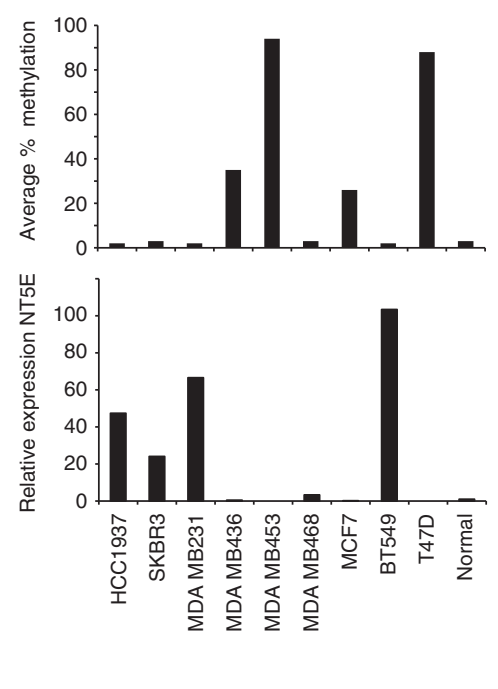

Figure 2 (A) Downregulation of NT5E mRNA correlates with CpG methylation in ER-positive breast carcinoma cell lines. Expression of NT5E mRNA was determined by qPCR and $\%$ methylation by pyrosequencing. Downregulation correlates with methylation. (B) NT5E mRNA is overexpressed in breast carcinoma cell lines lacking CpG island methylation. Average \% methylation in the NT5E CPG island was determined by pyrosequencing and expression of NT5E mRNA by qPCR (relative to normal breast epithelium). (C) Demethylation reactivates NT5E expression. MCF-7 cells were treated with AZA and harvested at the indicated times (in hours). Total RNA was isolated and levels of NT5E mRNA determined by GPCR as described in Materials and Methods.

NT5E CpG island) and downregulated relative to normal breast epithelium in MDA MB 436 and MCF-7 (partially methylated NT5E $\mathrm{CpG}$ island) (Figure 2A). Having confirmed correlation between methylation and downregulation of mRNA, we next analysed a larger panel of cell lines again using qPCR. NT5E mRNA was expressed at high levels in HCC1937 (BRCA1-associated TNBC), MDA MB 231 (TNBC), BT549 (TNBC) and SKBR3 (ER-, PgR - and HER-2 + ) (Figure 2B). All cell lines that overexpressed NT5E were unmethylated as determined by MSP and pyrosequencing. To further test the association of mRNA silencing with aberrant CpG methylation, we analysed the effect of the demethylating agent azacytidine ( $5^{\prime}$ AZA) in MCF-7 cells in which the $\mathrm{CpG}$ island was partially methylated and expression downregulated. Twenty-four hours after exposure to $5^{\prime}$ AZA there was an increase in NT5E mRNA levels and this increased further to a maximum three-fold upregulation after $96 \mathrm{~h}$ of $5^{\prime}$-AZA exposure (Figure 2C). Together, data from Figures 1 and 2 imply that the NT5E CpG island is unmethylated and the gene expressed at high levels predominantly but not exclusively in hormone receptor negative breast cancer cell lines, whereas, conversely, methylationdependent silencing of NT5E occurs more commonly in hormone receptor-positive cell lines.

\section{NT5E CpG island methylation associates with non-visceral predominant metastatic disease}

The in vitro data prompted us to investigate methylation in the NT5E $\mathrm{CpG}$ island in clinical cases of breast cancer. We first analysed using pyrosequencing DNA extracted from 83 formalin-fixed, paraffinembedded primary breast cancer cases from Northern Italy (Figure 3 ). These cases comprised 79 ER-positive cancers treated adjuvantly with endocrine therapy and 4 ER-negative cases. The NT5E CpG island was methylated in 40/79 (51\%) ER-positive cases. We asked

A
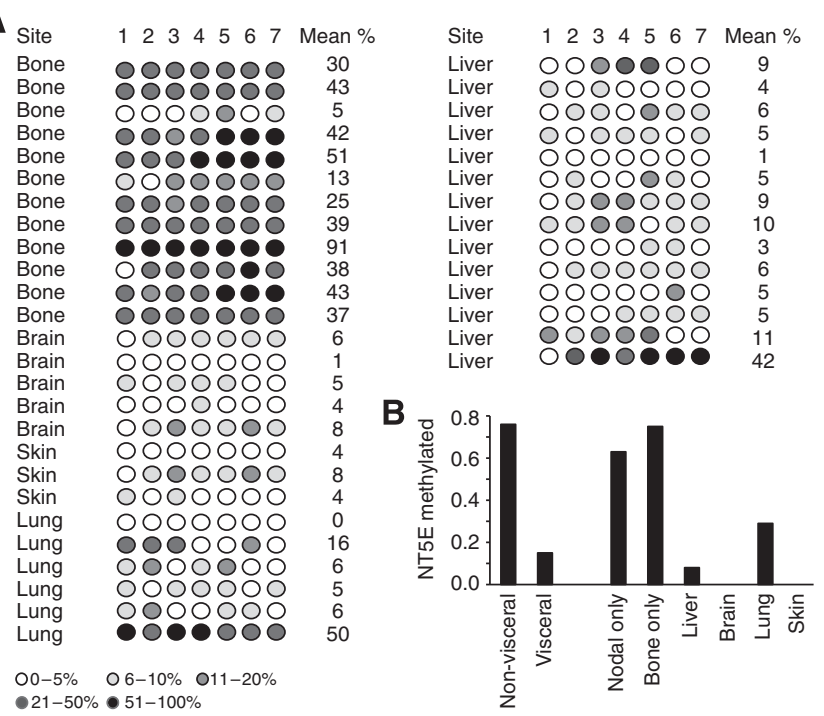

Figure 3 NT5E CPG island methylation is more common in cancers relapsing in non-visceral metastatic sites in ER-positive breast cancer (A) Representative pyrosequencing analysis of the NT5E CpG island in primary breast carcinomas. The predominant site of metastatic relapse is indicated. Pyrosequencing analysis was performed as described in Materials and Methods. The \% methylation at each analysed CpG dinucleotide is represented by the intensity of shading in the circles as shown in the figure, together with the mean \% methylation for each case. (B) Graph showing proportion of methylation-positive cases in ER-positive primary breast cancers, treated with endocrine therapy, which subsequently relapsed at different metastatic sites. Primary carcinomas which relapsed with metastasis to brain, liver, lung and skin are designated 'visceral'. Bone and lymph node metastasis are designated as 'non-visceral'.

whether methylation of the NT5E CpG island in primary ER-positive cases was associated with loco-regional and/or distant metastatic relapse. Representative pyrosequencing is shown in Figure $3 \mathrm{~A}$. The NT5E CpG island was densely methylated in 16 of $21(76 \%)$ patients who developed non-visceral metastatic disease compared with 6/28 cases with visceral metastases (21\%) $(P=0.0002, \mathrm{OR}=11.73)$. Interestingly, the NT5E CpG island was unmethylated in all four ER-negative cases and visceral metastatic relapse occurred in $3 / 4$ of these cases.

\section{NT5E CpG island methylation in metastatic breast cancer}

Data from primary cancers suggest that NT5E may influence the anatomical site of relapse. To further address this observation, we performed pyrosequencing analysis of 23 resected CNS metastases (series II) from patients with primary breast carcinomas. In each case, the diagnosis of metastatic breast cancer was confirmed by histopathological analysis and receptor expression studies (ER, PgR and HER-2). Of the 23 cases, 14 were ER negative and 8 of these 14 cases were TNBC. 10/14 ER-negative cases were negative for NT5E methylation and 6/8 TNBC were negative (Figure 4).

\section{Methylation in the NT5E CpG island varies with acquisition of a metastatic phenotype}

Our data imply selective pressure for overexpression of NT5E in at least a subset of cases of metastatic breast cancer, particularly in ER negative and TNBC cases. However, it is clear that some primary cancers, with high level of NT5E CpG island methylation, nonetheless ultimately relapsed in visceral sites. This prompted us to ask whether the methylation status of the NT5E CpG island changes in metastatic lesions relative to primary lesions. Using pyrosequencing, we analysed four cases in series II (all TNBC) for 
CpG $\begin{array}{llllllllll}1 & 2 & 3 & 4 & 5 & 6 & 7 & \text { Mean\% } & \text { ER PgR HER-2 }\end{array}$

Primary 1

Brain met 1

Primary 2

Brain met 2

Primary 3

Brain met 3

Primary 4

Brain met 4

Brain met

Brain met

Brain met

Brain met

Brain met

Brain met

Brain met

Brain met

Brain met

Brain met

Brain met

Brain met

Brain met

Brain met

Brain met

Brain met

Brain met

Brain met

Brain met

Normal breast

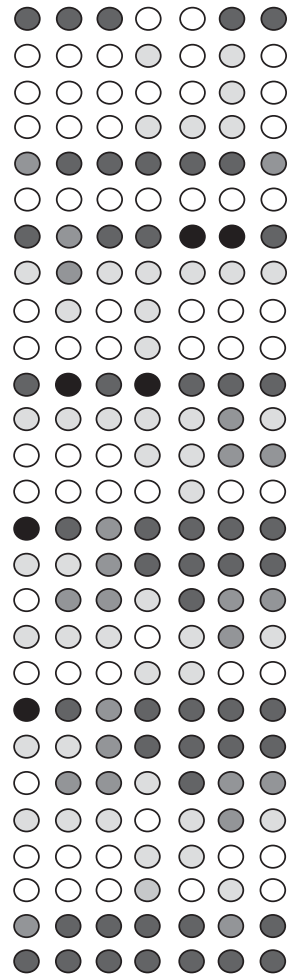$$
\begin{gathered}
18 \\
5 \\
2 \\
5 \\
22 \\
3 \\
38 \\
10 \\
2 \\
5 \\
79 \\
8 \\
7 \\
5 \\
34 \\
2 \\
14 \\
9 \\
5 \\
34 \\
21 \\
14 \\
9 \\
5 \\
2 \\
27 \\
45 \\
2
\end{gathered}
$$

○-5\% ○ $6-10 \% \bigcirc 11-20 \% \quad \bigcirc 21-50 \%$

Figure 4 Pyrosequencing analysis of NT5E CPG island in CNS metastatic breast cancer lesions. Pyrosequencing was done as described in Materials and Methods. The level of methylation is represented by the intensity of shading in the circles, each of which represents an individual CPG dinucleotide in the amplified fragment. The mean \% CpG methylation in the amplified fragment together with ER, PgR and Her-2 status of the lesion are indicated. Four cases of paired primary and CNS metastatic lesions are also shown to illustrate changes in methylation.

which paired primary and CNS metastatic tissue was available to us. There was no change in the status of ER, PgR or HER-2 between the primary and metastatic lesions in each case (Figure 4). Nevertheless in three cases of the CNS metastases there was a clear loss of methylation in the NT5E CpG island relative to the paired primary lesion, consistent with selection of cell clones lacking methylation (Figure 4). In the 4th case, methylation levels were low in the primary carcinoma and remained low in the CNS metastasis (Figure 4).

\section{NT5E CpG island methylation is an independent breast cancer biomarker}

The apparent association between the methylation status of the NT5E CpG island and the site specificity of metastasis prompted us to extend the investigation to an independent cohort of primary breast cancers, for which complete and long-term follow-up data were available. This series (series III) comprised 157 primary cancers cases (119 ER positive and 38 ER negative, of which 26 were TNBC; Table 2). Fifty-two patients had developed metastatic disease at the time of censoring. Methylation was detected in 96/ $157(61 \%)$ cases (Figure $5 \mathrm{~A})$, which is comparable to the first series analysed (51\%). Methylation was detected in both ER-positive $(77 / 119 ; 0.65)$ and ER-negative $(19 / 38 ; 0.5)$ cases. Patients whose primary tumours, were positive for NT5E CpG island methylation, were less likely to develop metastasis than those lacking
Table 2 (A) Clinico-pathological characteristics of patients $(n=157)$; (B) Multivariate analysis of prognostic factors

\begin{tabular}{|c|c|c|c|}
\hline Characterstic & & $\begin{array}{l}\text { Number of cases } \\
\text { (frequency) }\end{array}$ & \\
\hline \multicolumn{4}{|l|}{ (A) } \\
\hline \multicolumn{2}{|l|}{ Grade 1} & $29(0.19)$ & \\
\hline \multicolumn{2}{|l|}{ Grade 2} & $60(0.39)$ & \\
\hline \multicolumn{2}{|l|}{ Grade 3} & $63(0.4 I)$ & \\
\hline \multicolumn{2}{|l|}{ Missing } & 5 & \\
\hline \multicolumn{2}{|l|}{ Stage 1} & $63(0.4 I)$ & \\
\hline \multicolumn{2}{|l|}{ Stage 2} & $87(0.56)$ & \\
\hline \multicolumn{2}{|l|}{ Stage 3} & $6(0.03)$ & \\
\hline \multicolumn{2}{|l|}{ Missing } & & \\
\hline \multicolumn{2}{|l|}{ Node positive } & $69(0.45)$ & \\
\hline \multicolumn{2}{|l|}{ Node negative } & $83(0.55)$ & \\
\hline \multicolumn{2}{|l|}{ Missing } & 5 & \\
\hline \multirow{2}{*}{\multicolumn{2}{|c|}{$\begin{array}{l}\text { ER positive } \\
\text { ER negative }\end{array}$}} & $119(0.76)$ & \\
\hline & & $38(0.24)$ & \\
\hline \multirow{2}{*}{\multicolumn{2}{|c|}{$\begin{array}{l}\text { PgR positive } \\
\text { PgR negative }\end{array}$}} & $84(0.54)$ & \\
\hline & & $73(0.46)$ & \\
\hline \multicolumn{2}{|l|}{ HER-2 positive } & $19(0.12)$ & \\
\hline \multirow{2}{*}{\multicolumn{2}{|c|}{$\begin{array}{l}\text { HER-2 negative } \\
\text { Missing }\end{array}$}} & $136(0.88)$ & \\
\hline & & 2 & \\
\hline \multirow{2}{*}{\multicolumn{2}{|c|}{$\begin{array}{l}\text { TP53 mutation } \\
\text { TP53 wild-type }\end{array}$}} & $46(0.29)$ & \\
\hline & & $111(0.71)$ & \\
\hline \multirow{2}{*}{\multicolumn{2}{|c|}{$\begin{array}{l}\text { NT5E methylated (ER positive) } \\
\text { NT5E methylated (ER negative) }\end{array}$}} & $77(0.65)$ & \\
\hline & & $19(0.5)$ & \\
\hline \multicolumn{4}{|l|}{ (B) } \\
\hline Variable & Hazard ratio & $P$-value & $95 \% \mathrm{Cl}$ \\
\hline Tumour grade & 1.548 & 0.002 & $1.168-2.052$ \\
\hline Nodal status & 1.099 & 0.000 & $1.043-1.160$ \\
\hline ER positive & 0.372 & 0.12 & $0.107-1.291$ \\
\hline PgR positive & 0.497 & 0.182 & $0.178-1.387$ \\
\hline TP53 mutation & 2.041 & 0.08 & $0.919-4.529$ \\
\hline $\begin{array}{l}\text { NT5E } \\
\text { methylation }\end{array}$ & 0.328 & 0.010 & $0.141-0.765$ \\
\hline
\end{tabular}

methylation $(P=0.003, \mathrm{OR}=0.34,95 \%$ CI: $0.17-0.69)$. When analysing by histological subtype, we confirmed the association of $\mathrm{CpG}$ island methylation with lower probability of metastasis in ER negative $(P=0.049, \mathrm{OR}=0.21,95 \% \mathrm{CI}: 0.05-0.83)$ and in TNBC cases $(P=0.017, \mathrm{OR}=0.09,95 \% \mathrm{CI}$ : $0.01-0.55)$ (Figure $5 \mathrm{~B})$, but in the cohort of 119 ER-positive cases, this association just failed to reach statistical significance $(P=0.08, \quad O R=0.4271,95 \%$ CI: 0.20-1.09) (Figure 5B). When all cases were analysed, NT5E $\mathrm{CpG}$ island methylation was more common in those with non-visceral compared with visceral metastatic disease $(P=0.01$, $\mathrm{OR}=11.8)$. When analysing by histological subtype, this association was significant in ER-negative cases $(P=0.02, \mathrm{OR}=22)$, but did not reach significance in ER-positive cases $(P=0.33)$. Consistent with this observation, in cases with visceral metastases, the frequency of unmethylated NT5E CpG island was lower in ERpositive cases than in ER-negative cases (12/49 vs 12/19; $P=0.02$, $\mathrm{OR}=4.28)$. We then investigated NT5E CpG island methylation as a determinant of disease outcome in the same series, using timedependent endpoints. Patients with tumours lacking detectable NT5E CpG island methylation had increased probability of shorter DFS $(P=0.001, \mathrm{HR} 2.7$, Figure $5 \mathrm{C})$ and $\mathrm{OS}(P=0.001, \mathrm{HR}=3$, Figure 5D). In subgroup analysis, unmethylated NT5E CpG island was also associated with increased risk for poorer survival in ER 
A

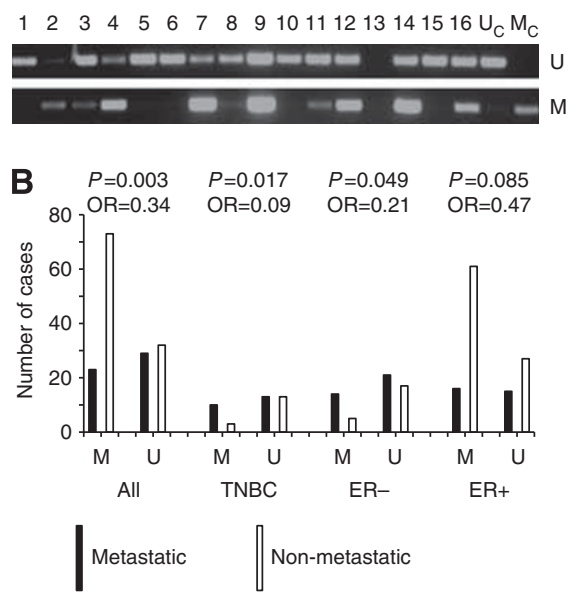

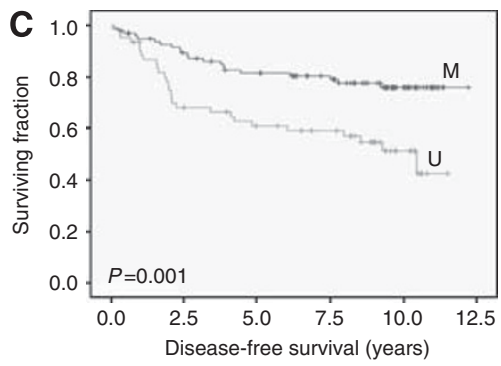

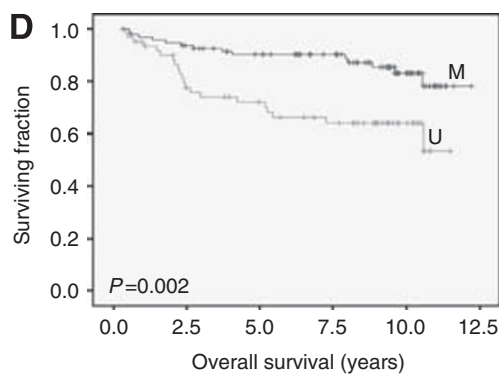

Figure 5 Methylation of the NT5E CPG island in primary breast carcinomas influences clinical outcome. (A) Representative methylation-specific PCR analysis of the NT5E CPG island in series III breast carcinomas. The figure shows unmethylated $(U)$ and methylated (M) reactions for each individual case DNA (numbered I-16). Also shown are control $U$ and M DNA samples modified in parallel with the cell line DNA samples. (B) Metastatic relapse is more frequent in patients whose primary breast carcinoma lacks methylation in the NT5E CpG island. The figure shows number of patients with metastatic relapse as a function of the methylation status of the NT5E CpG island $(M, U)$. All: $N=157$; TNBC: $N=26 ; E R-: N=38 ; E R+: N=119$. (C) Kaplan-Meir analysis of disease-free survival (DFS) in primary breast carcinomas according to methylation status of NT5E CpG island. Methylation in the NT5E CPG island was analysed by MSP and statistical analysis done as described in Materials and Methods. The figure shows DFS in primary cancers either M or U. (D) Kaplan-Meir analysis of OS in primary breast carcinomas according to methylation status of NT5E CpG island. Methylation in the NT5E CpG island was analysed by methylation-specific PCR and statistical analysis done as described in Materials and Methods. The figure shows OS in primary cancers either $\mathrm{M}$ or $\mathrm{U}$. Abbreviations: $\mathrm{ALL}=$ entire patient population; $\mathrm{ER}-=$ oestrogen receptor negative; $\mathrm{ER}+=$ oestrogen receptor positive; $\mathrm{MSP}=$ methylationspecific PCR; TNBC = triple-negative breast cancer.
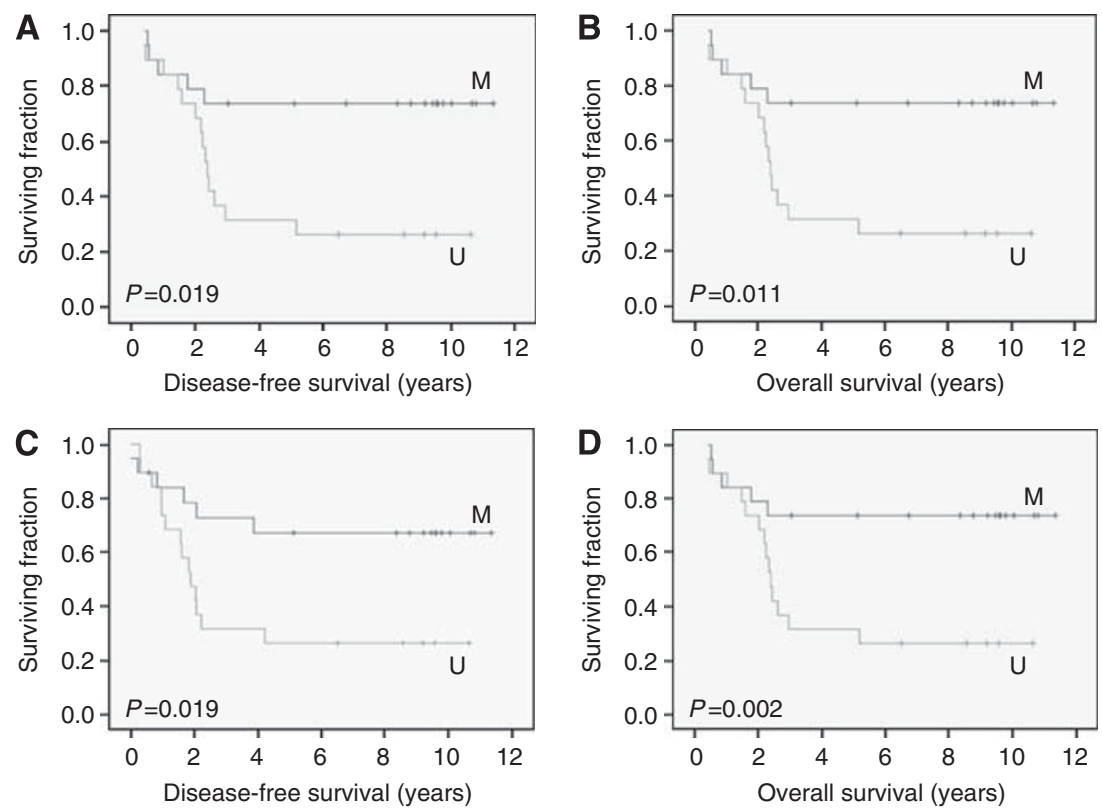

Figure 6 Kaplan-Meir analysis of outcome in ER negative and triple-negative primary breast carcinomas according to methylation status of NT5E CpG island. Methylation in the NT5E CpG island was analysed by methylation-specific PCR and statistical analysis done as described in Materials and Methods. The top two panels show DFS (A) and OS (B) in ER-negative primary cancers either methylated (M) or unmethylated (U) in the NT5E CpG island. The lower two panels show DFS $(\mathbf{C})$ and OS (D) in triple-negative breast carcinomas either M or $U$ in the NT5E CpG island.

negative $(P=0.011, \mathrm{HR}=3.27,95 \% \mathrm{CI}: 1.31-8.12$, Figure 6$)$ and in TNBC $(P=0.004$, HR $=6.2,95 \%$ CI: $1.9-20$ Figure 6$)$, but not in patients with ER-positive tumours $(P=0.35, \mathrm{HR}=1.64$,
95\% CI: 0.57-4.68). To further confirm that the effects of NT5E CpG island methylation are not the result of a non-specific 'methylator' phenotype, we tested the effects on clinical outcome of 
A
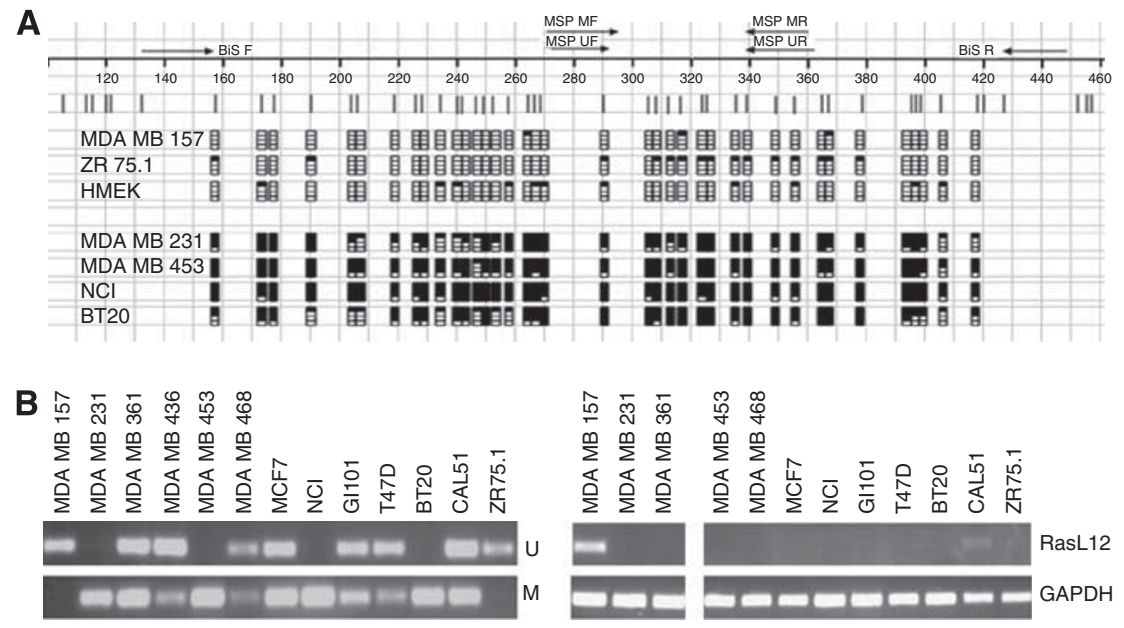

C

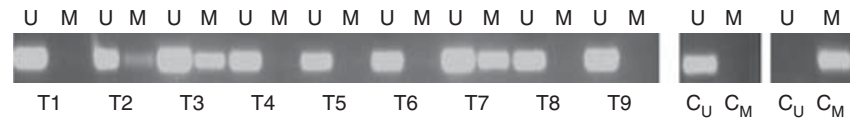

Figure 7 Methylation-dependent transcriptional silencing of RasL 12 in breast cancer. (A) Bisulphite sequencing analysis of Ras L 2 CpG island. The figure shows a map of the RasL 12 CpG island with the position of primers indicated for bisulphite sequencing (Bis F and Bis R) and methylation-specific PCR (MSP MF, MSP UF, MSP MR and MSP UR). Vertical lines below the scale represent individual CpG dinucleotides within the CpG island. The density of methylation for each cell line is represented by a quartile of blocks corresponding to each CpG. Black shading represents up to $25 \%$ methylation. Open blocks indicate no methylation. (B) Methylation-specific PCR analysis of RasL 12 CpG island methylation (left panel) and RT-PCR analysis of expression (right panel) in breast cancer cell lines. The figure shows $U$ - and M-specific PCR reactions for each cell line and RT-PCR. (C) MSP analysis of RasL 12 CpG island methylation in primary breast carcinomas from series III. Representative analysis of nine cases (TI-T9) is shown, together with control unmethylated ( $\left.\mathrm{C}_{U}\right)$ and control methylated $\left(C_{M}\right)$ DNA modified and analysed in parallel with the test samples.

a second novel epigenetically regulated gene, RasL12, in the same patient population. We initially showed using bisulphite sequencing and methylation-specific PCR that RasL12 is subject to CpG island methylation in many breast carcinoma cell lines, with a perfect correlation between bisulphite sequencing and methylation-specific PCR and downregulated expression of RasL12 (Figure 7A and Figure 7B). We then analysed the same patient population using methylation-specific PCR (Figure 7C). There was methylation in the RasL12 CpG island in 48/157 (31\%) cases. Methylation in the RasL12 CpG island did not significantly affect median DFS or median OS. For DFS: $P=0.5339, \mathrm{HR}=$ $0.7939,95 \%$ CI: $0.3836-1.643$; for OS: $P=0.3854, \mathrm{HR}=0.7608,95 \%$ CI: $0.4104-1.410$.

\section{NT5E CpG island methylation status and outcome after adjuvant chemotherapy}

We then asked whether the methylation status of NT5E affects clinical outcome in patients who received adjuvant chemotherapy. Disease-free survival in patients who received chemotherapy was significantly shorter in patients with unmethylated NT5E CpG island ( $P=0.0016, \mathrm{HR}=5.1,95 \% \mathrm{CI}$ : $1.8-14.37)$. Similarly, OS was shorter in cases with unmethylated NT5E CpG island $(P=0.0005$, $\mathrm{HR}=7.4,95 \%$ CI: 2.416-23.08).

\section{NT5E CpG island methylation is inversely correlated with TP53 mutation}

Finally, we performed multivariate analysis to assess the prognostic significance of NT5E CpG island methylation in series III (Table 2). This analysis revealed that NT5E methylation retained significance after multivariate analysis of known prognostic variables $(\mathrm{HR}=0.328,95 \% \mathrm{CI}: 0.141-0.765 ; P=0.010)$. Further, we found that NT5E CpG island methylation was inversely associated with TP53 mutation $(P<0.005)$.

\section{DISCUSSION}

Here, we report that NT5E (CD73) expression is regulated in breast cancer by the methylation status of the $\mathrm{CpG}$ island and that methylation is a prognostic indicator of favourable clinical outcome. Previous work has identified involvement of NT5E expression in cancer behaviour (Zhi et al, 2010), but this is to the best of our knowledge the first demonstration that NT5E is an independent prognostic marker in human cancer.

We have shown using two techniques, methylation-specific PCR and (quantitative) pyrosequencing, that downregulation of NT5E mRNA correlates well with methylation in the CpG island in breast cancer. In addition, we found that the NT5E CpG island is unmethylated and mRNA expressed in normal breast epithelial cells, while the hypomethylating compound $5^{\prime}$ AZA restores expression in breast cancer cell lines. Together, these observations imply that aberrant $\mathrm{CpG}$ methylation is an important mechanism of NT5E silencing in breast cancer and that silencing is specific for neoplasia. The expression analysis we report in breast cancer cell lines is consistent with previous work (Spychala et al, 2004; Mackay et al, 2009). We confirm and extend these expression analyses by showing that $\mathrm{CpG}$ island methylation is a key regulator of NT5E expression and the predominant mechanism of downregulation in breast cancer cell lines. Our results also further extend previous data by showing that expression in ER-positive (predominantly luminal) breast carcinoma cell lines is downregulated by methylation-dependent transcriptional silencing and that in the absence of methylation (most commonly, but not 
exclusively, seen in ER negative and triple-negative cell lines), NT5E is often greatly overexpressed.

To assess the clinical significance of our cell line observations, we extended the study to primary breast carcinomas. Given the potential importance of NT5E as a mediator of malignant progression and metastasis, studies of NT5E expression in clinical cases of breast cancer have been remarkably limited. One such study, performed 20 years ago, employed immunohistochemical analysis of NT5E in an unselected series of breast carcinomas and showed that expression was detected in $\sim 10 \%$ (Kruger et al, 1991). More recently, it was shown in a small series of clinical cases that NT5E was underexpressed predominantly in ER-positive breast carcinomas and more commonly overexpressed in TNBC (Spychala et al, 2004). Our data, in three independent series of breast carcinomas, are clearly consistent with both previous studies. Furthermore, we show in a series of ER-positive cases treated with adjuvant endocrine therapy that bone predominant disease, a clinical entity with a typically indolent clinical course (Andrade et al, 2011), is more common in cases with dense NT5E CpG island methylation, whereas visceral and CNS metastases predominate in cases lacking dense NT5E methylation. We confirmed these results in a second-independent clinical series containing ER positive, ER negative and TNBC. Aggressive disease, with visceral and/or brain metastases, is more likely in cases that lack methylation in the NT5E CpG island, than in those with methylation. Our data are consistent with the observation that overexpression of NT5E in an experimental model of breast cancer promotes metastasis to the lungs via activation of $\mathrm{A} 2 \mathrm{~B}$ adenosine receptors (Stagg et al, 2011).

Despite this strong propensity for cases lacking methylation to metastasise to viscera and brain, it is clear that (in a minority of cases) clinically aggressive disease develops from primary breast cancers in which the NT5E promoter CpG island is methylated. We propose two potential explanations for this. First, knock-down of NT5E causes increased cell migration in some cell types (Andrade et al, 2011). It is therefore possible that, perhaps in early disease, methylation-dependent silencing of NT5E might promote enhanced migration in a subset of breast carcinomas through as yet undefined mechanisms. Second, we have shown in the present study that the methylation status of the NT5E CpG island often changes from methylated to unmethylated in cases which have metastasised to brain, when compared with the primary breast carcinoma from which they derived, implying a strong selective pressure favouring (re-) expression of NT5E in aggressive breast cancers. The numbers of such paired cases available to us was limited. However, to the best of our knowledge, this is the first demonstration of dynamic epigenetic change in a specific gene from primary carcinoma to brain metastasis in breast cancer. Changes in expression of steroid hormone receptors and HER-2 between primary and recurrent disease are now well described (Aitken et al, 2010; Thompson et al, 2010) and changes in the epigenetic status of NT5E provide further evidence of a dynamic transcriptional process underlying metastasis.

To validate methylation in NT5E as an independent prognostic marker, we tested a second novel epigenetically regulated gene, RasL12. We showed using bisulphite sequencing and methylationspecific PCR that expression of RasL12 is silenced by methylation in breast carcinoma cell lines and in primary breast carcinomas. Understanding of the precise role of RasL12 in breast cancer awaits additional studies. However, the absence of any predictive effect of RasL12 on clinical outcomes in our series of cases strongly endorses the specific biomarker candidacy of NT5E methylation.

In addition to influencing metastatic potential in breast cancer, we demonstrate that the methylation status of NT5E also affects outcome in cases treated with (predominantly anthracycline) chemotherapy. These results are supported by previous work in cell lines resistant to anthracyclines (Ujhazy et al, 1994; Mikhailov et al, 2008). Depletion of NT5E mediated by inhibitory RNA causes increased apoptosis and reduction in cell growth rate in MDA MB 231 cells consistent with the clinical importance of NT5E CpG island methylation in TNBC and particularly with its strong predictability of favourable outcome to adjuvant chemotherapy, potentially by intervening in accumulation of intracellular nucleotides that have been shown to act as critical pro-survival factors (Chandra et al, 2006). A further potential mechanism linking absence of methylation with less favourable outcome after chemotherapy is the highly significant association between absence of NT5E CPG island methylation and mutation in TP53.

The recognised role of NT5E in promoting invasion and metastasis (Wang et al, 2008) taken together with the frequent absence of methylation in NT5E in ER negative and TNBC, which we demonstrate in the present work, implies that inhibition of NT5E may be an attractive therapeutic strategy in these subtypes of breast cancer. Strategies to inhibit or block NT5E clearly merit consideration in breast cancer particularly in the difficult to treat TNBC and ER-negative cases. Conversely, our results may have implications in the potential use of demethylating agents in breast (and other) solid tumours. This approach has been considered because the majority of genes affected by methylation-dependent transcriptional silencing in cancer are tumour suppressor genes or genes silenced by methylation in cancers with acquired chemotherapy resistance. Re-expression of such genes would have likely therapeutic value. Our data imply that reactivation of NT5E would have deleterious effects by promoting a more aggressive phenotype and potentially resistance to chemotherapy. Identification of patients likely to benefit from use of demethylating agents might be informed, at least in part, by profiling of the methylation status of key genes, such as NT5E.

In conclusion, we show for the first time that NT5E is regulated epigenetically in breast cancer, the epigenetic status of this gene influencing metastasis and clinical outcome. We suggest that NT5E promoter $\mathrm{CpG}$ island methylation may serve as a promising favourable breast cancer epigenetic biomarker. NT5E is expressed in normal breast tissue (http://www.proteinatlas.org/ ENSG00000135318). As such, the sensitivity of methylation analysis and its specificity for neoplasia together suggest that NT5E methylation analysis may be more informative and clinically useful than immunohistochemistry or $\mathrm{qPCR}$ as a prognostic biomarker. Our results warrant independent confirmation by other groups before considering its investigation as a metastasis predictor in clinical trials of adjuvant breast cancer therapies.

\section{ACKNOWLEDGEMENTS}

The work was supported by the Brain Tumour Research Charity (BTRC), Cancer Research-UK, Breast Cancer Research (Scotland) and the Tayside Tissue Bank. Eleftheria Hatzimichael received funding as a scholar from the Hellenic Society of Haematology Foundation. Carlo Palmieri is the recipient of a Cancer Research UK Clinician Scientist award. Ondrej Gojis is in part funded by a grant from the Ministry of Education of the Czech Republic (Project 'Oncology' MSM 0021620808) and is also a recipient of the Translational Research Fellowship from the European Society of Medical Oncology and a fellowship from the European Society for Surgical Oncology. The Division of Cancer at Imperial College London, Imperial College Healthcare NHS Trust is an Experimental Cancer Medicine Centre (ECMC) that is supported by funds from Cancer Research UK and the Department of Health (C37/A7283) and also forms part of the Imperial Cancer Research UK Centre (C42671/A12196). Tim Crook is a Scottish Senior Clinical Fellow. We thank F Roncaroli for his contribution. 


\section{REFERENCES}

Aitken SJ, Thomas JS, Langdon SP, Harrison DJ, Faratian D (2010) Quantitative analysis of changes in ER, PR and HER2 expression in primary breast cancer and paired nodal metastases. Ann Oncol 21(6): 1254-1261

Andrade CM, Lopez PL, Noronha BT, Wink MR, Borojevic R, Margis R, Lenz G, Battastini AM, Guma FC (2011) Ecto-5'-nucleotidase/CD73 knockdown increases cell migration and mRNA level of collagen I in a hepatic stellate cell line. Cell Tissue Res 344(2): 279-286

Andreopoulou E, Hortobagyi GN (2008) Prognostic factors in metastatic breast cancer: successes and challenges toward individualized therapy. J Clin Oncol 26(22): 3660-3662

Boyle JM, Hey Y, Guerts van Kessel A, Fox M (1988) Assignment of ecto5'-nucleotidase to human chromosome 6. Hum Genet 81(1): 88-92

Chandra D, Bratton SB, Person MD, Tian Y, Martin AG, Ayres M, Fearnhead HO, Gandhi V, Tang DG (2006) Intracellular nucleotides act as critical prosurvival factors by binding to cytochrome $\mathrm{C}$ and inhibiting apoptosome. Cell 125(7): 1333-1346

Jaenisch R, Bird A (2003) Epigenetic regulation of gene expression: how the genome integrates intrinsic and environmental signals. Nat Genet 33(Suppl): 245-254

Korde LA, Gralow JR (2011) Can We Predict Who's at Risk for Developing Bone Metastases in Breast Cancer? J Clin Oncol 29(27): 3600-3604

Kruger KH, Thompson LF, Kaufmann M, Moller P (1991) Expression of ecto-5'-nucleotidase (CD73) in normal mammary gland and in breast carcinoma. Br J Cancer 63(1): 114-118

Lopez J, Percharde M, Coley HM, Webb A, Crook T (2009) The context and potential of epigenetics in oncology. Br J Cancer 100(4): 571-577

Mackay A, Tamber N, Fenwick K, Iravani M, Grigoriadis A, Dexter T, Lord CJ, Reis-Filho JS, Ashworth A (2009) A high-resolution integrated analysis of genetic and expression profiles of breast cancer cell lines. Breast Cancer Res Treat 118(3): 481-498

McGuire WL (1991) Breast cancer prognostic factors: evaluation guidelines. J Natl Cancer Inst 83(3): 154-155

Mikhailov A, Sokolovskaya A, Yegutkin GG, Amdahl H, West A, Yagita H, Lahesmaa R, Thompson LF, Jalkanen S, Blokhin D, Eriksson JE (2008) CD73 participates in cellular multiresistance program and protects against TRAIL-induced apoptosis. J Immunol 181(1): 464-475

Paik S, Shak S, Tang G, Kim C, Baker J, Cronin M, Baehner FL, Walker MG, Watson D, Park T, Hiller W, Fisher ER, Wickerham DL, Bryant J, Wolmark N (2004) A multigene assay to predict recurrence of tamoxifentreated, node-negative breast cancer. N Engl J Med 351(27): 2817-2826

Perez EA (2011) Breast cancer management: opportunities and barriers to an individualized approach. Oncologist 16(Suppl 1): 20-22

Perou CM, Sorlie T, Eisen MB, van de Rijn M, Jeffrey SS, Rees CA, Pollack JR, Ross DT, Johnsen H, Akslen LA, Fluge O, Pergamenschikov A, Williams C, Zhu SX, Lonning PE, Borresen-Dale AL, Brown PO, Botstein D (2000) Molecular portraits of human breast tumours. Nature 406(6797): 747-752
Roukos DH, Murray S, Briasoulis E (2007) Molecular genetic tools shape a roadmap towards a more accurate prognostic prediction and personalized management of cancer. Cancer Biol Ther 6(3): 308-312

Shah R, Smith P, Purdie C, Quinlan P, Baker L, Aman P, Thompson AM, Crook T (2009) The prolyl 3-hydroxylases $\mathrm{P} 3 \mathrm{H} 2$ and $\mathrm{P} 3 \mathrm{H} 3$ are novel targets for epigenetic silencing in breast cancer. Br J Cancer 100(10): 1687-1696

Sotiriou C, Neo SY, McShane LM, Korn EL, Long PM, Jazaeri A, Martiat P, Fox SB, Harris AL, Liu ET (2003) Breast cancer classification and prognosis based on gene expression profiles from a population-based study. Proc Natl Acad Sci USA 100(18): 10393-10398

Sotiriou C, Pusztai L (2009) Gene-expression signatures in breast cancer. $N$ Engl J Med 360(8): 790-800

Spychala J, Lazarowski E, Ostapkowicz A, Ayscue LH, Jin A, Mitchell BS (2004) Role of estrogen receptor in the regulation of ecto-5'-nucleotidase and adenosine in breast cancer. Clin Cancer Res 10(2): 708-717

Stagg J, Divisekera U, Duret H, Sparwasser T, Teng MW, Darcy PK, Smyth MJ (2011) CD73-deficient mice have increased antitumor immunity and are resistant to experimental metastasis. Cancer Res 71(8): 2892-2900

Thompson AM, Jordan LB, Quinlan P, Anderson E, Skene A, Dewar JA, Purdie CA (2010) Prospective comparison of switches in biomarker status between primary and recurrent breast cancer: the Breast Recurrence In Tissues Study (BRITS). Breast Cancer Res 12(6): R92

Ujhazy P, Berleth ES, Pietkiewicz JM, Kitano H, Skaar JR, Ehrke MJ, Mihich E (1996) Evidence for the involvement of ecto-5'-nucleotidase (CD73) in drug resistance. Int J Cancer 68(4): 493-500

Ujhazy P, Klobusicka M, Babusikova O, Strausbauch P, Mihich E, Ehrke MJ (1994) Ecto-5'-nucleotidase (CD73) in multidrug-resistant cell lines generated by doxorubicin. Int J Cancer 59(1): 83-93

Visvanathan K, Sukumar S, Davidson NE (2006) Epigenetic biomarkers and breast cancer: cause for optimism. Clin Cancer Res 12(22): 6591-6593

Wang L, Zhou X, Zhou T, Ma D, Chen S, Zhi X, Yin L, Shao Z, Ou Z, Zhou P (2008) Ecto-5'-nucleotidase promotes invasion, migration and adhesion of human breast cancer cells. J Cancer Res Clin Oncol 134(3): 365-372

Zhi X, Wang Y, Zhou X, Yu J, Jian R, Tang S, Yin L, Zhou P (2010) RNAimediated CD73 suppression induces apoptosis and cell-cycle arrest in human breast cancer cells. Cancer Sci 101(12): 2561-2569

Zhou P, Zhi X, Zhou T, Chen S, Li X, Wang L, Yin L, Shao Z, Ou Z (2007a) Overexpression of Ecto-5'-nucleotidase (CD73) promotes T-47D human breast cancer cells invasion and adhesion to extracellular matrix. Cancer Biol Ther 6(3): 426-431

Zhou X, Zhi X, Zhou P, Chen S, Zhao F, Shao Z, Ou Z, Yin L (2007b) Effects of ecto-5'-nucleotidase on human breast cancer cell growth in vitro and in vivo. Oncol Rep 17(6): 1341-1346

Zimmermann H (1992) 5'-Nucleotidase: molecular structure and functional aspects. Biochem J 285(Pt 2): 345-365

This work is published under the standard license to publish agreement. After 12 months the work will become freely available and the license terms will switch to a Creative Commons Attribution-NonCommercial-Share Alike 3.0 Unported License. 